\title{
Molecular demographic history of the Hainan Peacock Pheasant (Polyplectron katsumatae) and its conservation implications
}

\author{
CHANG Jiang ${ }^{1,2}$, CHEN De $^{1}$, LIANG Wei $^{3}$, LI Ming $^{2} \&$ ZHANG ZhengWang ${ }^{1 *}$ \\ ${ }^{1}$ MOE Key Laboratory for Biodiversity Science and Ecological Engineering, College of Life Sciences, Beijing Normal University, \\ Beijing 100875, China; \\ ${ }^{2}$ Key Laboratory of Animal Ecology and Conservation Biology, Institute of Zoology, Chinese Academy of Sciences, Beijing 100101, China; \\ ${ }^{3}$ MOE Key Laboratory for Tropical Animal and Plant Ecology, College of Life Sciences, Hainan Normal University, Haikou 571158, China
}

Received July 18, 2012; accepted July 31, 2012; published online January 16, 2013

\begin{abstract}
Knowledge of the historical responses of animal species to climate changes is critical for understanding their evolutionary history and conservation. During the late Quaternary period, Southeast Asia had a larger land area than today due to lower sea levels, and its terrestrial landscape was covered by extensive forests and savannah. To date, however, the general fluctuations in landscape distribution and their impacts on the demographics history of native species during the late Quaternary periods are still disputed. Specifically, the responses of animals on Hainan Island, which is located in the northernmost region of Southeast Asia, to historical climate changes, are poorly understood. Here, we performed a series of demographic analyses based on mitochondrial DNA genes to examine the response of the resident Hainan Peacock Pheasant (Polyplectron katsumatae) to climate change. Unlike the pattern of population collapse during the ice age and expansion during the warming period, we detected a historical expansion pattern in the demographic history of Hainan Peacock Pheasant through the late Quaternary period. It was concluded that the Hainan Peacock Pheasant survived through the late Quaternary periods, despite of its currently limited distribution and population size on Hainan Island. Anthropogenic influences must be considered in conservation planning due to their impacts on currently fragmented habitats and populations.
\end{abstract}

phylogeography, Quaternary, Hainan Island, Polyplectron katsumatae, conservation

Citation: Chang J, Chen D, Liang W, et al. Molecular demographic history of the Hainan Peacock Pheasant (Polyplectron katsumatae) and its conservation implications. Chin Sci Bull, 2013, 58: 2185-2190, doi: 10.1007/s11434-012-5629-6

The Pleistocene climatic changes have left notable footprints in the demographic history of most Northern Hemisphere species [1]. In Europe and North America, species contracted and retreated southwards to climatic refugia during glacial-interglacial cycles and expanded northwards, dispersing into their current distribution areas during the warming periods $[1,2]$. Owing not only to the high level of diversity of wildlife but also to the richness of endemic species, regions of Southeast Asia are recognized as global biodiversity hotspots and are focal regions for ecologists and conservation biologists [3,4]. To date, the influence of Quaternary glaciations on molecular diversity and the demographic history of species in these areas remain poorly

*Corresponding author (email: zzw@bnu.edu.cn) understood, especially for species on Hainan Island, which lies in the northernmost region of Southeast Asia. In the tropical mountains of Southeast Asia, there was no large-scale continental glaciation during the Quaternary period [1]. It has been proposed that tropical mountains provided a stable, moist habitat through the Quaternary period, which allowed suitable habitats and lineages to survive through altitudinal shifts [1]. However, during the Last Glacial Maximum (LGM), with the advent of glaciations, the mean annual temperature dropped an estimated $4-6^{\circ} \mathrm{C}$, and the climate became drier, which reduced the global sea level and exposed the continental shelf throughout Southeast Asia [5]. This, taken together with the genetic uniqueness of faunas on Hainan Island (such as Polyplectron katsumatae) and the deep genetic divergence from their relatives on the 
mainland of China (such as Polyplectron bicalcaratum) [6], makes it reasonable to postulate that within the Pleistocene period, faunas on Hainan Island underwent demographic fluctuations as well. By using marine and terrestrial pollen diagrams for paleoclimate research, both Hope et al. [7] and Yan [8] suggested that during the later part of the Quaternary period, such as in the LGM and the Holocene, Hainan Island has experienced long-term changes in climate and vegetation, which could have affected the habitat in which wildlife was distributed.

Hainan Island $\left(18^{\circ} 09^{\prime}-20^{\circ} 10^{\prime} \mathrm{N}, 108^{\circ} 23^{\prime}-111^{\circ} 03^{\prime} \mathrm{E}\right)$ is one of the biodiversity hotspots in China $[4,9]$. The island, consisting of $33900 \mathrm{~km}^{2}$, is separated from mainland China by the Qiongzhou Strait, which is $\sim 20 \mathrm{~km}$ wide and $100 \mathrm{~m}$ deep. Yan [8] indicated that Hainan Island experienced the process of repeated connection with and disconnection from the mainland throughout the Pleistocene, as global sea levels repeatedly rose and fell [10]. During the LGM, the sea level fell by $120-150 \mathrm{~m}$ in the South China Sea [11]. Therefore, Hainan Island represents a typical continental land-bridge island and provides opportunities for exploring the responses of wildlife to climate change. Assessing the demographic history of island species also provides insight into the genetic background and requirements for conservation of vulnerable species [12]. However, the understanding of how wildlife on Hainan Island responded to climate change remains poor. One major reason for this lack of understanding could be the severe anthropogenic contraction of endemic wildlife on the island [13,14].

The Hainan Peacock Pheasant (Polyplectron katsumatae), which is endemic to Hainan island $[15,16]$, has been recognized as a distinct species [6] and is listed as an Endangered (EN) species by the IUCN Red List since 2010 [17]. In China, it was listed as an endangered bird within Category I of nationally protected animals [18]. The Hainan Peacock Pheasant is currently restricted to fragmented evergreen and semi-evergreen areas in the mountainous region of central and southwestern Hainan Island at 600-1200 m, and they may also live in mature secondary forests $[17,19]$. These habitats are under considerable anthropogenic threat, and by the late $1980 \mathrm{~s}$, tropical forest cover was reduced to no more than $3000 \mathrm{~km}^{2}$ on Hainan Island [20]. In addition to habitat loss and degradation, illegal hunting was thought to have played a significant role in population declines [17,21]. However, the molecular demographic history of the $P$. katsumatae on Hainan Island remains poorly understood. In addition, knowledge of the molecular profiles of endangered species is crucial for creating promising conservation strategies and maintaining extant populations [22]. Therefore, in this study, multiple fragments of mitochondrial DNA (mtDNA) from individuals throughout the entire distribution areas on Hainan Island were used to explore how Pleistocene climate fluctuations affected Hainan Island species through the examination of the demographic history of $P$. katsumatae; then provide conservation suggestions of the endangered species.

\section{Materials and methods}

\subsection{Sample collection, DNA extraction, PCR amplifica- tion and sequencing}

The samples (feather and blood) were collected from 42 individuals throughout Hainan Island. Total DNA was extracted with the TIANamp genomic DNA extraction kit (TIANGEN, China). Polymerase chain reaction (PCR) amplification was performed, with primers for whole mitochondrial cytochrome b gene (cytb) and NADH dehydrogenase subunit 2 gene (ND2) as described by Chang [23]. The PCR products were sequenced on a 3730 DNA sequencer (Applied BioSystems, USA). The sequences were visually compared to their original chromatograms to avoid reading errors and then checked with online published DNA data. To avoid amplifying mitochondrial DNA homologues from the nuclear genome (numts), we verified the sequences in the following manner: (1) designed specific primers, including those amplifying longer segments of mtDNA ( $>1$ $\mathrm{kb})$; (2) checked for the presence of PCR ghost bands, or extra bands; (3) detected the presence of sequence ambiguities, frameshift mutation and stop codons; (4) used BLAST to do a sequence similarity search and alignment with the published sequence databases of related species that were deposited in GenBank; (5) detected whether unexpected phylogenetic placements existed using a phylogeny analysis; and (6) detected whether the base composition of mtDNA in this bird is companied by a deficiency of $\mathrm{G}$ and $\mathrm{T}$ and an excess of $\mathrm{A}$ and $\mathrm{C}$, and rates of codon substitution is order of third position $>$ first position $>$ second position.

\subsection{MtDNA data analysis}

The complete ND2 and cytb sequences were obtained by aligning the partial sequences with the software SeqEdit (Applied Biosystems Inc., USA). Two single amplifications were performed. As the ND2 and $c y t b$ sequences were uniform in length, the alignment was straightforward. The nucleotide diversity and haplotype diversity were estimated by DNASP 5.0 [24]. The phylogenetic relationships among haplotypes were constructed by using maximum parsimony implemented in NETWORK 4.5.1.6 [25]. We applied several methods to test the population fluctuation of the Hainan Peacock Pheasant. First, Tajima's D [26] and Fu's Fs tests [27] were carried out in MEGA 5 [28]. We also used grey peacock pheasant (Polyplectron bicalcaratum) as outgroup for calculating Fay and Wu's $H$ value [29]. Fay and Wu's $H$ measures departures from neutrality between intermediate-frequency and high-frequency alleles [29]. In contrast, $D$ measures departures from neutrality in the difference between intermediate frequency and low-frequency alleles. Thus, it is helpful for us to combine both tests to distinguish 
population expansion from selection. Second, the program ARLEQUIN 3.5 [30] which could perform mismatch distribution analysis was applied to detect whether a population experienced expansion event. Specifically, the relationship $\tau=2 u t$, where $u=\mu k$ ( $\mu$ is the substitution rate per nucleotide and $k$ is the number of nucleotides) was used to calculated the timing of population expansion. The $1 \%$ substitution rate per million years was taken as the molecular clock of mitochondrial DNA genes [31]. Third, the demographic history was detected with the Bayesian skyline plot (BSP) implemented in the BEAST 1.5.4 [32]. The convergent check was determined using three independent Markov Chain Monte Carlo (MCMC) runs of $5 \times 10^{8}$ steps, with $10 \%$ burn-in and displayed using Tracer 1.4 (http://beast.bio.ed. ac.uk/Tracer). In addition, the rates of codon substitution of mtDNA genes were estimated in BEAST 1.5.4.

\section{Results and discussion}

\subsection{Molecular diversity and demographic history of $P$. katsumatae}

The obtained sequences were authentic mtDNA and not from nuclear mitochondrial-origin sequences (numts) for they did not contain stop codons and deletions/insertions, and the base composition of mtDNA genes is companied by a deficiency of $\mathrm{G}(11.7 \%)$ and $\mathrm{T}(24.4 \%)$ and an excess of A $(28.3 \%)$ and $\mathrm{C}(35.6 \%)$, and varied codon substitution rates is order of third position $>$ first position $>$ second position (GenBank number: JQ917212-21). Of the complete 1041 bp of ND2 and 1143 bp of $c y t b$ sequences from 42 individuals of $P$. katsumatae, we obtained eight haplotypes with combined sequences defined by eleven variable sites. The combined haplotype diversity was 0.78 , and the nucleotide diversity was 0.0007 . In this case, $P$. katsumatae currently retains lower mtDNA nucleotide diversity and smaller population size compared with other pheasant species [33] and its mainland relative $P$. bicalcaratum (unpublished data). The pattern of low mtDNA genetic diversity was consistent with other Hainan faunas [13,14,34]. To date, it has been deduced that anthropogenic activities could exert a catastrophic effect on species on Hainan Island [13-15, 35,36].

The results of Tajima's D $(-1.72, P>0.05)$ and Fu's $F s$ $(-2.97, P<0.05)$ was negative, and Fay and Wu's $H$ was not significant $(0.12, P>0.05)$. In general, a negative value of $D$ or $F s$ implied that a recent population expansion or the selection, while non-significant value of $H$ could be recognized as the indicator of the population expansion event instead of the selection. Due to $F s$ is more sensitive than $D$ in detecting the population expansion and genetic hitchhiking, it is normal that we obtained a negative significant Fu's $F s$ value but a negative non-significant Tajima's $D$. In addition, mismatch (unimodal figure) and network analysis (star-like) also provide consistent results of the population expansion event (Figures 1 and 2). It is accepted that populations which have experienced a demographic expansion event $[37,38]$ or though a range expansion with migrations between neighboring sites $[39,40]$ should display a unimodal curve of mismatch analysis. Specifically, the estimated timing of the population expansion event was about 28-53 ka before present (ka BP), which falling into the time of Marine Isotope Stage 3 (MIS 3). In addition, according to the BSP result, a pattern of historical population size growth during the late Quaternary period (about 30-50 ka BP) was also concluded, which implies that the pheasant survived during the LGM even with a certain of population growth (Figure 3).

In conclusion, historical population expansion has been detected for P. katsumatae on Hainan Island, and the starting time of expansion was in the period of MIS 3. In contrast to MIS 4 and MIS 2 periods, MIS 3, a period between 60-27 ka BP during the last glacial cycle, experienced several abrupt climatic warming phases. Some studies have shown that the hydrothermal condition of MIS 3 was much better than that of MIS 2 and MIS 4, and even reached an
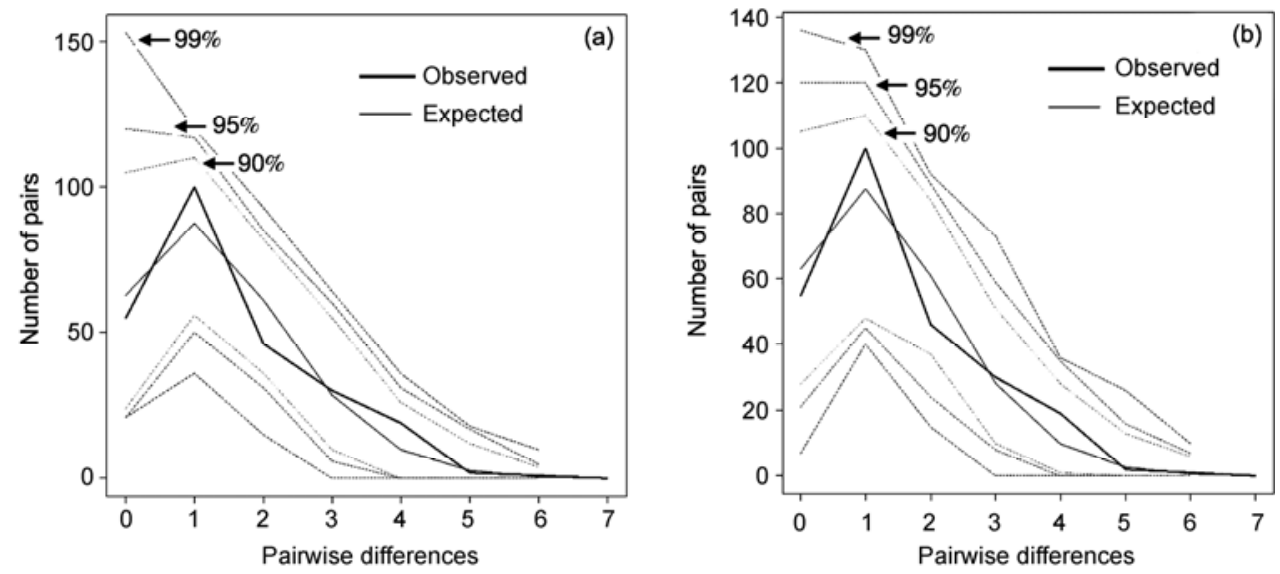

Figure 1 Mismatch distribution of expected and observed with dotted lines of different confidence intervals. (a) Spatial expansion and (b) demographic expansion of $P$. katsumatae. On the horizontal axis is the number of nucleotide site differences between pairs of individuals. 


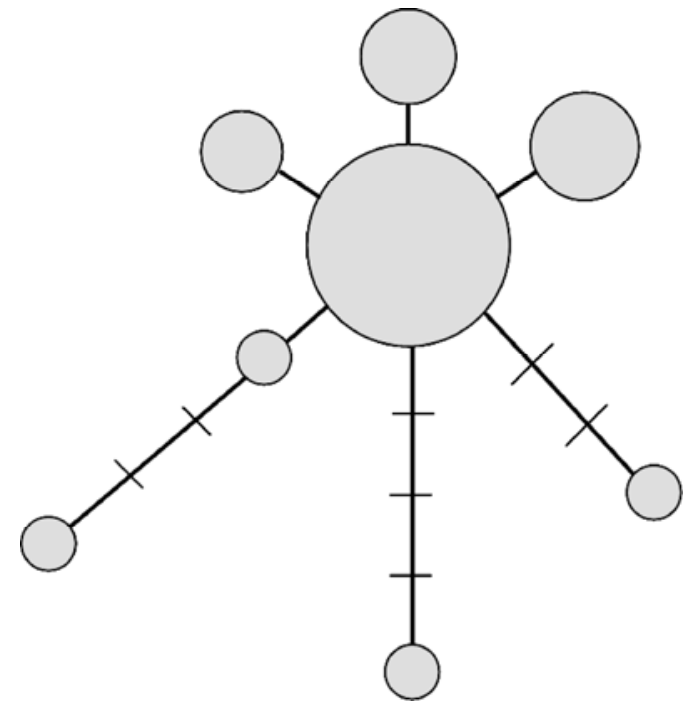

Figure 2 Unrooted haplotype network analysis of $P$. katsumatae based on $c y t b$ and $N D 2$ sequences. Each circle represents a unique haplotype. The size of the round indicates the number of each haplotype. Each intersecting line represents a single mutational change according to length.

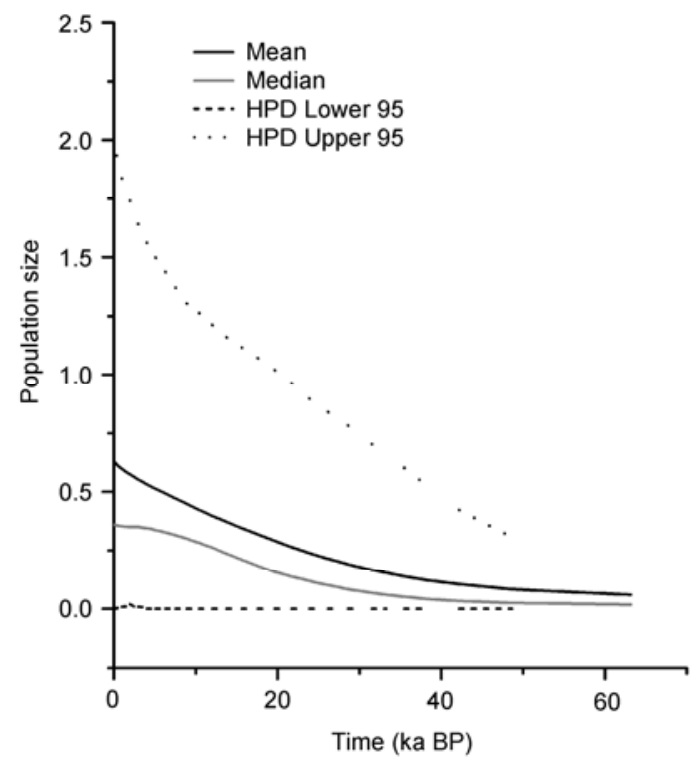

Figure 3 Bayesian skyline plot of effective population size (Ne $\mu$ ) through time. Median estimates are shown by the solid grey line, along with the union of the $95 \%$ highest posterior density (HPD) areas in dot lines.

interglacial climate condition similar to MIS 5 [41]. Shi et al. [42] named this period the High Temperature and Large Precipitation Event. Therefore, it is not unusual that the population expansion of $P$. katsumatae occurred from MIS 3. However, the following reasons might contribute to the pattern of historical population expansion even during the LGM. Shen et al. [43] showed that precipitation in northeastern China in MIS 3a was 100-400 mm higher than during the present period. More evidences have been found that a wet period once occurred in MIS 3 in Southeast Asia [44]. Therefore, it was recognized that the abrupt climatic trans- gression that occurred 60-50 ka BP, and peaked around 40-30 ka BP, was controlled by enhanced precipitation rather than temperature [42]. Because P. katsumatae had coped with the colder weather during the LGM, we then deduced that this species might be more susceptible to precipitation than temperature. Besides of the long-term climatic evolution, short-term repeated water-level fluctuations might also contribute to the population expansion of $P$. katsumatae during the LGM. Some studies have previously indicated that a broad, continuous, lowland tropical rainforest and savannah over major portions of Sundaland were maintained by lower sea levels during the LGM, and might contribute to the unusual population expansion during the LGM [45-48]. Cannon et al. [46] have modeled the distribution changes of Sundaland rainforests during the last 120000 year glacial cycles, finding that the rainforests experienced unusual expansion rather than contraction in their ranges. They proposed that it is today's rainforests that are refugial and not those of, for example, the LGM. To date, some findings suggest that animal populations extend their distribution to cope with the LGM, as supported by pollen analysis of river and offshore sediments from the South China Sea $[49,50]$. Hainan Island has experienced connections and disconnections with the mainland of China, Taiwan Island and the Indo-China Peninsula due to sea level fluctuations throughout the late Pleistocene [10]. Therefore, the potential expansion of the land areas and vegetation of Hainan Island could also contribute to the survival of $P$. katsumatae through the LGM. This is also supported by the spatial expansion detected with mismatch analysis.

\subsection{Conservation implications}

Because many extant species in Southeast Asia once have survived multiple ice age's cycles since the Pleistocene, they will probably be less susceptible by temperature than the length of the dry-season and seasonality [42]. For example, it was indicated that populations of lowland Siamese fireback pheasant Lophura diardi have experienced expansion over 25 years in Thailand [51]. In addition, Peh [52] described that some birds have expanded their upper limits along elevation gradients. Unfortunately, much suitable habitats and habitat corridors are vanishing caused by recent anthropogenic environmental changes, such as habitat loss and fragmentation. Probabilities for range expansion are now hampered in the current islands of Southeast Asia, sustaining populations in situ is becoming the only option [47]. Currently, conservationists are paying more attention to in situ small and fragmented populations of species, as these are the ones at the greatest risk of extirpation [53-59]. Specifically, small isolated populations are sensitive to genetic erosion. The losing of genetic diversity by chance and by inbreeding will contribute to the extirpation of species and populations. This is also true for $P$. katsumatae on Hainan Island, where recent anthropogenic activities have affected 
the habitat connectivity of $P$. katsumatae. Actually, field work using radio-tracking data has detected that the home range of the $P$. katsumatae was quite small and relatively fixed [17]. Till now, the pheasant distributed only in the evergreen broadleaf-dominated forests of mountainous region in central and southwestern of Hainan Island [17].

During the past several decades, populations of $P$. katsumatae have suffered threats, such as overexploitation for timber and the destruction for hunting [17]. Deforestation and replacement with exotic tree species such as Acacia mangium, and Eucalyptus spp. occurred in some forest areas, and these are important threats to the Hainan Peacock Pheasant and other forest birds, e.g. Hainan partridge (Arborophila ardens) [17,53]. The illegal hunting of birds is found in most surveyed areas and is carried out mainly for sale in markets [60]. The finding that the Hainan Peacock Pheasant was not occurred in any of the forest plantations, and the facts of hunting this peacock pheasant was common, indirectly indicates that the anthropogenic activities might have greatly reduced its numbers [17]. Actually, it has been reported the population of the Hainan Peacock Pheasant had declined from approximately 2700 in 1990 [61] to only approximately 300 individuals in 2000 [62] and is at risk of losing the ability to be self-sustainable.

The findings of the study may contribute to improving management practices for the conservation of this endemic pheasant. Our results demonstrated that the historical population of P. katsumatae has expanded since the late Quaternary, and even survived through the LGM. Unfortunately, the current severe habitat fragmentation and small population size [17] and potential inbreeding risks must be imputed to anthropogenic factors. Therefore, conservation efforts should be made to avoid anthropogenic declines in Hainan Peacock Pheasant populations and potential inbreeding by sustaining populations in place without disturbance.

We thank Wang Biao, Yang Canchao and Cai Yan for their help in sampling and data collection. This work was supported by the National Natural Science Foundation of China-Guangdong Joint Fund (U0833005), China Postdoctoral Science Foundation (2012M510543), the National Natural Science Foundation of China (30360015 and 30860044), and the Program for New Century Excellent Talents in University (NCET-10-0111). We thank the Forestry Department of Hainan Province, Hainan Bawangling and Yinggeling Nature Reserves for support and permission to collect samples. We also thank Dr. Shouhsien Li and two anonymous reviewers for helpful comments and suggestions.

1 Hewitt G M. The genetic legacy of the Quaternary ice ages. Nature, 2000, 405: 907-913

2 Zink R M. Comparative phylogeography in North American birds. Evolution, 1996, 50: 308-317

3 Myers N, Mittermaier R A, Mittermeier C G, et al. Biodiversity hotspots for conservation priorities. Nature, 2000, 403: 853-858

4 Sodhi N S, Koh L P, Brook B W, et al. Southeast Asian biodiversity: An impending disaster. Trends Ecol Evol, 2004, 19: 654-660

5 Bintanja R, van de Wal R S W, Oerlemans J. Modelled atmospheric temperatures and global sea levels over the past million years. Nature, 2005, 437: $125-128$
6 Chang J, Wang B, Zhang Y Y, et al. Molecular evidence for species status of the endangered Hainan peacock pheasant. Zool Sci, 2008, 25: $30-35$

7 Hope G, Kershaw A P, Kaars S V D, et al. History of vegetation and habitat change in the Austral-Asianregion. Quatern Int, 2004, 118119: 103-126

8 Yan J A. Paleontology and ecologic environmental evolution of the Quaternary in Hainan Island. J Palaeogeogr, 2006, 8: 103-115

9 Tang Z Y, Wang Z Y, Zheng C Y, et al. Biodiversity in China's mountains. Front Ecol Environ, 2006, 4: 347-352

10 Hope G. The Quaternary in Southeast Asia. In: Gupta A, ed. The Physical Geography of Southeast Asia. Oxford: Oxford University Press, 2005

11 Wang P. The ice-age China Sea-research results and problems. In: Proceedings of the First International Conference on Asian Marine Geology. Beijing: China Ocean Press, 1990. 181-197

12 Hedrick P W. Genetics of Populations, 2nd ed. Boston: Jones and Bartlett, 2000

13 Pang J F, Hoelze R, Song Y L, et al. Lack of mtDNA control region variation in Hainan Eld's deer: Consequence of recent population bottleneck? Conserv Genet, 2003, 4: 109-112

14 Monda K, Simmons R E, Kressirer P, et al. Mitochondrial DNA hypervariable region-1 sequence variation and phylogeny of the concolor gibbons, Nomascus. Am J Primatol, 2007, 69: 1-22

15 Cheng T H. A Synopsis of the Avifauna of China. Beijing: Sciences Press, 1987

16 Zheng G M. A Checklist on the Classification and Distribution of the Birds of China. Beijing: Science Press, 2005

17 Liang W, Zhang Z W. Hainan Peacock Pheasant (Polyplectron katsumatae): An endangered and rare tropical forest bird. Chin Birds, 2011, 2: 111-116

18 Zheng G M, Wang Q S. China Red Data Book of Endangered Animals (Aves). Beijing: Science Press, 1998

19 Madge S, McGowan P. Pheasants, Partridges and Grouse. London: Christopher Helm, 2002

20 Lin M Z, Zhang Y L. Dynamic changes of tropical forest in Hainan Island (in Chinese). Geogr Res, 2001, 20: 703-712

21 Liang W, Cai Y W, Yang C C. Extreme levels of hunting of birds in a remote village of Hainan Island, China. Bird Conserv Int, 2012, doi: 10.1017/S0959270911000499

22 Crandall K A, Bininda-Emonds O R P, Mace G M, et al. Considering evolutionary processes in conservation biology. Trends Ecol Evol, 2009, 17: 390-395

23 Chang J. Using molecular techniques to improve the understanding and conservation of the endangered species: Examples from four endemic Galliformes species of China. Dissertation for the Doctoral Degree. Beijing: Beijing Normal University, 2010

24 Librado P, Rozas J. DnaSP v5: A software for comprehensive analysis of DNA polymorphism data. Bioinformatics, 2009, 25: 1451-1452

25 Bandelt H J, Forster P, Röhl A. Median-joining networks for inferring intraspecific phylogenies. Mol Biol Evol, 1999, 16: 37-48

26 Tajima F. Statistical method for testing the neutral mutation hypothesis by DNA polymorphism. Genetics, 1989, 123: 585-595

27 Fu Y X. Statistical tests of neutrality of mutations against population growth, hitchhiking and background selection. Genetics, 1997, 147: 915-925

28 Tamura K, Peterson D, Peterson N, et al. MEGA5: Molecular evolutionary genetics analysis using maximum likelihood, evolutionary distance, and maximum parsimony methods. Mol Biol Evol, 2011, 28: 2731-2739

29 Fay J C, Wu C I. Hitchhiking under positive Darwinian selection. Genetics, 2000, 155: 1405-1413

30 Excoffier L, Lischer H E L. Arlequin 3.5: A new series of programs to perform population genetics analyses under Linux and Windows. Mol Ecol Res, 2010, 10: 564-567

31 Lovette I J. Mitochondrial dating and mixed support for the "rule" in birds. Auk, 2004, 121: 1-6

32 Rambaut A, Drummond A J. BEAST: Bayesian evolutionary analysis by sampling trees. BMC Evol Biol, 2007, 1: 214 
33 Liu Y, Zhan X J, Wang N, et al. Effect of geological vicariance on mitochondrial DNA differentiation in Common Pheasant populations of the Loess Plateau and eastern China. Mol Phyl Evol, 2010, 55: 409-417

34 Zou F, Lim H C, Marks B D, et al. Molecular phylogenetic analysis of the Grey-cheeked Fulvetta (Alcippe morrisonia) of China and Indochina: A case of remarkable genetic divergence in a "species". Mol Phylogenet Evol, 2007, 44: 165-174

35 Hao S D, Huang W B. Luobidong Cave Site of Sanya (in Chinese). Haikou: Nan Fang Publishing House, 1998. 1-122

36 Zhang M, Fellowes J R, Jiang X, et al. Degradation of tropical forest in Hainan, China, 1991-2008: Conservation implications for Hainan Gibbon (Nomascus hainanus). Biol Conserv, 2010, 143: 1397-1404

37 Rogers A R, Harpending H. Population growth makes waves in the distribution of pairwise genetic differences. Mol Biol Evol, 1992, 9: 552-569

38 Slatkin M, Hudson R R. Pairwise comparisons of mitochondrial DNA sequences in stable and exponentially growing populations. Genetics, 1991, 129: 555-562

39 Ray N, Currat M, Excoffier L. Intra-deme molecular diversity in spatially expanding populations. Mol Biol Evol, 2003, 20: 76-86

40 Excoffier L. Patterns of DNA sequence diversity and genetic structure after a range expansion: lessons from the infinite-island model. Mol Ecol, 2004, 13: 853-864

41 Shi Y F, Zheng B X, Su Z. The Quaternary Glaciations and Environmental Variations in China. Shijiazhuang: Hebei Scientific Press, 2006

42 Shi Y F, Liu X D, Li B Y, et al. A very strong summer monsoon event during 30-40 ka BP in the Qinghai-Xizang (Tibet) Plateau and its relation to precessional cycle (in Chinese). Chin Sci Bull (Chin Ver), 1999, 14: 1475-1480

43 Shen H Y, Zhang Y M, Jia Y L. An organic carbon isotopic record from sediment of the Huangqihai lake, Inner Mongolia: Implications of environmental evolution (in Chinese). Mar Geol Quat Geol, 2005, 25: $35-40$

44 Wang X M, Sun X J, Wang P X, et al. The records of coastline changes reflected by mangroves on the Sunda Shelf since the last 40 ka. Chin Sci Bull, 2008, 53: 2069-2076

45 Bird M I, Taylor D, Hunt C. Palaeoenvironments of insular Southeast Asia during the Last Glacial Period: A savanna corridor in Sundaland? Quatern Sci Rev, 2005, 24: 2228-2242

46 Cannon C H, Morley R J, Bush A B G. The current refugial rainforests of Sundaland are unrepresentative of their biogeographic past and highly vulnerable to disturbance. Proc Natl Acad Sci USA, 2009, 106: 11188-11193

47 Woodruff D S. Biogeography and conservation in Southeast Asia: How 2.7 million years of repeated environmental fluctuations affect today's patterns and the future of the remaining refugial-phase biodi- versity. Biodivers Conserv, 2010, 1-23

48 Wurster C M, Bird M I, Bull I D, et al. Forest contraction in north equatorial Southeast Asia during the Last Glacial Period. Proc Natl Acad Sci USA, 2010, 107: 15508-15511

49 Sun X, Li X, Luo Y, et al. The vegetation and climate at the last glaciation on the emerged continental shelf of the South China Sea. Palaeogeogr Palaeoclimatol Palaeoecol, 2000, 160: 301-316

50 Wang X M, Sun X J, Wang P X, et al. Vegetation on the Sunda Shelf, South China Sea, during the Last Glacial Maximum. Palaeogeogr Palaeoclimatol Palaeoecol, 2009, 278: 88-97

51 Round P D, Gale G A. Changes in the status of Lophura pheasants in Khao Yai National Park, Thailand: A response to warming climate? Biotropica, 2008, 40: 225-230

52 Peh K S H. Potential effects of climate change on elevational distributions of tropical birds in Southeast Asia. Condor, 2007, 109: 437-441

53 Sexton J P, McIntyre P J, Angert A L, et al. Evolution and ecology of species range limits. Annu Rev Ecol Evol Syst, 2009, 40: 415-436

54 Fang S G. Does population increase alone ensure the long-term survival of endangered species? Chin Sci Bull, 2011, 56: 2521-2522

55 He G, Huang K, Guo S T, et al. Evaluating the reliability of microsatellite genotyping from low-quality DNA templates with a polynomial distribution model. Chin Sci Bull, 2011, 56: 2523-2530

56 Dai Q, Fu J Z. When central populations exhibit more genetic diversity than peripheral populations: A simulation study. Chin Sci Bull, 2011, 56: 2531-2540

57 Pan H J, Shi F L, Chang Z F, et al. Mitochondrial DNA variation analysis suggests extreme low genetic diversity in Guizhou snubnosed monkeys (Rhinopithecus brelichi). Chin Sci Bull, 2011, 56: 2541-2544

58 Shi L, Wang R W, Zhu L X, et al. Varying coefficient analysis for indeterminate species interactions with non-parametric estimation, exemplifying with a fig-fig wasp system. Chin Sci Bull, 2011, 56: 2545-2552

59 Xiang Z F, Yu Y, Yang M, et al. Does flagship species tourism benefit conservation? A case study of the golden snub-nosed monkey in Shennongjia National Nature Reserve. Chin Sci Bull, 2011, 56: 2553-2558

60 Liang W, Wang J C, Su W B, et al. Surveys of the Hainan Partridge Arborophila ardens on Hainan Island, China. In: Fuller R A, Browne S J, eds. Galliformes 2004. Proceedings of the 3rd International Galliformes Symposium. World Pheasant Association, Fordingbridge, UK, 2005

61 Gao Y R, Yu D Q. Hainan peacock pheasant (in Chinese). Chin J Zool, 1990, 25: 42-44

62 China State Forestry Administration. Reports of National Wildlife Surveys of China (in Chinese). Beijing: China Forestry Publishing House, 2009

Open Access This article is distributed under the terms of the Creative Commons Attribution License which permits any use, distribution, and reproduction in any medium, provided the original author(s) and source are credited. 